\title{
Déterminants Individuels Et Contextuels Du Paludisme Chez Les Enfants De 6-59 Mois En République Démocratique Du Congo
}

\author{
Christian Ngomenzey Musuyi \\ Franklin Bouba Djourdebbe \\ Emmanuel Ekambi \\ Institut de Formation et de Recherche Démographiques, \\ Université de Yaoundé II SOA
}

Doi:10.19044/esj.2021.v17n7p94

Submitted: 03 December 2020

Accepted: 22 January 2021

Published: 28 February 2021
Copyright 2021 Author(s)

Under Creative Commons BY-NC-ND 4.0 OPEN ACCESS

Cite As:

Ngomenzey Musuyi C., Bouba Djourdebbe F. \& Ekambi E. (2021). Déterminants Individuels Et Contextuels Du Paludisme Chez Les Enfants De 6-59 Mois En République Démocratique Du Congo. European Scientific Journal, ESJ, 17(7), 94.

https://doi.org/10.19044/esj.2021.v17n7p94

\section{Resumen}

Le paludisme est une maladie parasitaire qui sévit principalement dans les pays pauvres de la zone tropicale. Parallèlement à la recherche d'un vaccin, la politique préventive contre le paludisme reste l'utilisation de la moustiquaire en RDC tout comme dans d'autres pays africains. Cependant, le taux de prévalence du paludisme chez les enfants de moins de cinq ans a augmenté comparativement à son niveau de 2014. Cette étude vise une meilleure compréhension des déterminants du paludisme chez les enfants de 6-59 mois en RDC. Les données utilisées proviennent de l'Enquête Démographique et de Santé (EDS) de 2013-2014. Deux méthodes sont utilisées dans ce travail : l'analyse descriptive (bivariée et multivariée) et l'analyse explicative multiniveau. En premier lieu, à travers l'analyse factorielle des correspondances multiples, ce travail a montré que les enfants impaludés résident en milieu rural et dans les provinces du Centre, du Nordest et du Nord-ouest. En général, ces enfants vivent dans les communautés où la proportion des pauvres est élevée. Ils appartiennent à des ménages pauvres qui ne disposent d'aucune moustiquaire, de ce fait, ces enfants ne dorment pas sous une moustiquaire. Leurs mères sont agricultrices et sans niveau 
d'instruction ou de niveau primaire. En second lieu, à travers une régression logistique multiniveau, ce travail a montré que les enfants qui vivent dans les provinces du Centre et du Sud-est contractent davantage le paludisme que ceux de l'Ouest. Le risque de contracter le paludisme baisse avec l'amélioration du niveau de vie des ménages et l'amélioration du niveau d'instruction de la mère. Par ailleurs, ce travail a identifié la promiscuité dans le ménage, le type de lieu d'aisance, l'utilisation de la moustiquaire, l'âge de l'enfant comme les déterminantes du paludisme chez les enfants en RDC. Cette étude a permis de mettre en lumière les différents déterminants qui modulent la transmission du paludisme. Ce constat devrait se traduire par une réadaptation des stratégies de lutte contre le paludisme en RDC. Ces stratégies de lutte devraient cibler les enfants de 24-59 mois en tenant compte de la disparité régionale.

Mots clés : Enfant, Environnement, Paludisme, Analyse multiniveau, RDC

\title{
Individual and Contextual Determinants of Malaria in Children 6-59 Months in the Democratic Republic of the Congo
}

\author{
Christian Ngomenzey Musuyi \\ Franklin Bouba Djourdebbe \\ Emmanuel Ekambi \\ Institute for Training and Demographic Research, \\ University of Yaoundé II SOA
}

\begin{abstract}
Malaria is a parasitic disease that rages mainly in poor countries in the tropics. Along with the search for a vaccine, the preventive policy against malaria remains the use of mosquito nets in the DRC as in other African countries. However, the prevalence rate of malaria in children under five has increased compared to 2014 levels. This study aims to better understand the determinants of malaria in 6-59 months in the DRC. The data used comes from the 2013-2014 Demographic Health and Survey (DHS). Two methods are used in this work: descriptive analysis (bivariate and multivariate) and multilevel explanatory analysis. First, through the factorial analysis of multiple correspondents, this work has shown that malarious children reside in rural areas and in the Center, North-East and North-West provinces. In general, these children live in communities where the proportion of poor people is high. They belong to poor households that do not have any mosquito
\end{abstract}


nets, so these children do not sleep under the mosquito net. Their mothers are farmers and have no education or primary education. Secondly, through the multi-level explanatory analysis, this work has shown that children living in the central and south-eastern provinces contract malaria more than those in the west. The risk of contracting malaria decreases as the standard of living of households improves and the level of education of the mother improves. In addition, this work identified the promiscuity in the household, the type of toilet facility, the use of the mosquito net, the age of the child as the determining factors of malaria in children in the DRC. This study has made it possible to shed light on the various factors which modulate the transmission of malaria. This observation should result in a readaptation of control strategies against malaria in DRC. These control strategies should target children aged 24-59 months, taking into account the regional disparity.

Keywords: Child, Environment, Malaria, Multilevel analysis, DRC

\section{Introduction}

Les débats sur les facteurs explicatifs du paludisme et les politiques de lutte ne cessent de s'amplifier en dépit des travaux déjà réalisés dans des disciplines diverses et variées (Pierrat, 2010). Des mécanismes restent en effet à comprendre afin de tenter de juguler cet enjeu de santé publique majeur pour les pays du Sud (Moiroux, 2012). Le paludisme est une maladie parasitaire qui sévit principalement dans les pays pauvres de la zone tropicale (Saïdou, 2018 ; Soma et al., 2018). Il est dû à un protozoaire du genre plasmodium transmis à l'homme par la piqûre d'un moustique du genre Anophèle femelle infectée (Carnevale et Robert, 2009). Cette infection palustre constitue un risque majeur de morbidité et de mortalité (Audry et Gaüzère, 2019). En 2010, près de la moitié de la population mondiale était exposée aux risques de contracter le paludisme (Hanitriniaina, 2017). L'Organisation Mondiale de la Santé (OMS) fait état de 219 millions de cas et 435000 décès rapportés dans le monde au cours de l'année 2017 (OMS, 2018). Les enfants de moins de cinq ans ont représenté $61 \%$ (266 000) des décès dus au paludisme dans le monde (OMS, 2018).

De toutes les régions affectées par cette maladie, l'Afrique supporte une part disproportionnée de la charge mondiale du paludisme. En 2016, 9 cas de paludisme sur 10 et 9 décès sur 10 dus à cette maladie sont survenus sur ce continent. Environ $80 \%$ de cas du paludisme proviennent de quatorze pays de l'Afrique subsaharienne et de l'Inde. Près de la moitié des cas enregistrés en 2017 dans le monde concerne quatre pays de l'Afrique subsaharienne à savoir le Nigéria (25\%), la République Démocratique du Congo (RDC) (11\%), le Mozambique (5\%) et l'Ouganda (4\%) (OMS, 2018). En RDC, le paludisme demeure l'endémie majeure et la première cause de morbidité (Losimba et al., 
2018). Il est également parmi les trois premières causes de mortalité dans le groupe des plus vulnérables, à savoir les enfants de moins de cinq ans et les femmes enceintes (MPSMRM, MSP et ICF International, 2014). En plus des pertes en vies humaines et de l'absentéisme scolaire, le paludisme coûte cher en dépenses de santé publique. Un épisode de paludisme grave revient à au moins 95 \$ US pour l'enfant, sachant qu'un enfant congolais fait, en moyenne, 10 épisodes de fièvre par an (MPSMRM, MSP et ICF International, 2014). Le paludisme est un facteur d'aggravation de la pauvreté, une cause d'inégalités et un frein au développement (Saïdou, 2018).

Pourtant, les Objectifs de Développement Durable (ODD) ${ }^{1}$, la déclaration d'Alma-Ata $(1978)^{2}$, la déclaration d'Abuja ${ }^{3}$ et l'initiative mondiale «Faire Reculer le Paludisme » $(\mathrm{FRP})^{4}$ mettent l'accent sur la réduction de la morbidité et de la mortalité dues au paludisme. La reconnaissance de l'importance de ce problème sanitaire et de l'existence de ces disparités a donné lieu aux à plusieurs études sur les déterminants du paludisme. Outre leurs apports scientifiques, elles ont joué un rôle important dans l'élaboration des stratégies de lutte contre ce problème de santé publique. Certes, ces études ont porté des fruits, mais ceux-ci restent insuffisants car le problème demeure préoccupant dans la plupart des pays africains (OMS, 2018). Il existe une hétérogénéité spatiale et sociale module différemment dans la transmission du paludisme (Remy, 1988 ; Maud, 2006 ; Nkemba et al., 2014). Les travaux de plusieurs auteurs ont mis en exergue l'influence des facteurs contextuels dans le risque d'attraper le paludisme (Noguer, 1979 ; Pierrat, 2010). Or, les modèles multiniveaux ont été développés pour répondre aux problèmes spécifiques posés par des données structurées selon plusieurs niveaux (Courgeau et Baccaïni, 1997 ; Nganawara, 2016). Les enfants d'un même quartier ou d'un même ménage partagent un ensemble de caractéristiques communes qui peuvent contribuer à la survenue du paludisme (Koné, 2012). Les unes de ces caractéristiques étant attachées à l'aléa et les autres à la vulnérabilité. Les politiques de préventions contre les moustiques

${ }^{1}$ L'Objectif de Développement Durable (ODD3) relatif à la santé. Particulièrement la cible 3. 3 «D'ici à 2030, mettre fin à l'épidémie du VIH/Sida, à la tuberculose, au paludisme et aux maladies tropicales négligées et combattre l'hépatite, les maladies transmises par l'eau et autres maladies transmissibles $»$.

${ }^{2}$ Un des objectifs de la déclaration d'Alma-Ata se rapportait à la prévention et le contrôle des grandes endémies contre le paludisme.

${ }^{3}$ Un des objectifs du sommet des chefs d'états et de gouvernements africains tenu à Abuja était qu'en 2005, au moins $60 \%$ des enfants atteints de paludisme auront accès à un traitement approprié dans les 24 heures.

${ }^{4}$ L'initiative mondiale FRP (Roll Back Malaria, RBM en Anglais) s'est fixée comme objectif la réduction de la mortalité spécifique liée au paludisme, de $50 \%$ d'ici 2010 , de $30 \%$ d'ici 2015 et de $20 \%$ d'ici 2025. En l'an 2030, le paludisme devrait, ainsi, cesser d'être une cause majeure de morbidité, de mortalité et de pertes socioéconomiques en Afrique. 
se fondent sur les facteurs liés à la vulnérabilité que sur ceux qui se rattachent à l'aléa (Pierrat, 2010).

La première conception du paludisme au II ${ }^{\text {ime }}$ siècle avant Jésus-Christ se fonde sur la corrélation entre la présence d'eau stagnante, les marais et les fièvres bénignes à effets pathogènes (Stefani, 2011). Ainsi, deux moyens de protection étaient mis en exergue : éviter d'habiter les zones inondables insalubres et drainer les marais (Drame, 2010). La théorie de transmission vectorielle du paludisme par des moustiques émise par Ross et Manson en 1897 a conduit aux politiques de destruction des moustiques (Capelle, 2007). Déjà dans les années 1960, l'échec de la politique d'éradication des moustiques au $\mathrm{DDT}^{5}$ a été suivi par l'émergence de la résistance de l'anophèle aux insecticides et la résistance du parasite aux antipaludiques (Pierrat, 2010). Parallèlement à la poursuite des recherches sur un potentiel vaccin et des nouveaux traitements, la prévention est au centre de la gestion de l'épidémie (Pierrat, 2010; OMS, 2015). Le principal moyen de prévention reste la moustiquaire (Mamadou, 2018). Certaines études montrent que l'utilisation de moustiquaire réduit fortement le risque d'attraper le paludisme (Takudzwa, 2018 ; Hajison et al., 2018). Comme dans d'autres pays africains, la stratégie préventive de lutte contre le vecteur du paludisme en RDC est essentiellement l'utilisation de la moustiquaire (PNLP-RDC, 2017).

Cependant entre 2014 et 2018, le taux d'utilisation de la moustiquaire chez les enfants de moins de cinq ans est passé de 56\% à 51\% (MPSMRM, MSP et ICF International, 2014 ; INS-RDC, 2018). A côté de cette baisse légère de l'utilisation de la moustiquaire, une augmentation de la prévalence du paludisme chez les enfants de moins de cinq ans de $23 \%$ à $31 \%$ s'observe (MPSMRM, MSP et ICF International, 2014 ; INS-RDC, 2018). D'une part, ce constat semble montrer que l'utilisation de la moustiquaire n'est pas l'unique variable à prendre en compte dans la prévention du paludisme. D'autre part, ce constat évoque la nécessité de rechercher d'autres variables déterminantes dans l'explication du paludisme chez l'enfant. Cet article vise à (i) dresser le profil des enfants de 6-59 mois par rapport au paludisme et à (ii) identifier les déterminants individuels et contextuels du paludisme chez les enfants de 6-59 mois.

\section{Méthodes}

\section{Contexte d'étude et données}

La RDC est un pays d'Afrique centrale. Elle se situe à cheval sur l'Equateur avec une superficie de $2345409 \mathrm{~km} 2$. De plus, elle a une ligne côtière de 42 kilomètres de long sur l'océan atlantique à l'embouchure du

\footnotetext{
${ }^{5}$ DDT : Dichlorodiphényltrichloroéthane, premier insecticide moderne développé au début de la seconde guerre mondiale. Le DDT entraînait une forte pollution des sols.
} 
fleuve Congo (MPSMRM, MSP et ICF International, 2014). La ligne côtière peut créer des biotopes favorables au développement des gîtes larvaires.

La pluviométrie annuelle en RDC est généralement élevée. Elle varie entre 1500 et $2000 \mathrm{~mm}$ près de l'équateur, et entre 1400 et $1500 \mathrm{~mm}$ dans les zones tropicales et montagneuses (MPSMRM, MSP et ICF International, 2014). Cette pluviométrie est à la base de multiples crues et du développement de nombreux marécages, gîtes potentiels d'anophèles. La pyramide d'âge de la RDC a une base très large qui se rétrécie rapidement vers un sommet effilé, ce qui traduit un niveau de fécondité. Par conséquent, la taille des ménages est élevée, particulièrement en milieu urbain où $17 \%$ des ménages comptent 9 personnes (MPSMRM, MSP et ICF International, 2014). Pourtant, une taille réduite de la famille permet d'augmenter les investissements réalisés en matière de santé, particulièrement dans la prévention contre le paludisme, pour chaque enfant (Banque Mondiale, 2018).

La persistance de la violence et de l'insécurité a déchiré le tissu social du pays. Plus de 3,5 millions de personnes ont perdu la vie dans la guerre qui a éclaté en 1998, dont près de la moitié était des enfants de 6-59 mois (Banque Mondiale, 2018). Dans les provinces de l'Est, environ 2 millions d'enfants n'ont pas accès à l'éducation ni à la santé, car les conflits ont endommagé les écoles et les FOSA. Dans un contexte d'instabilité politique et/ou de crise, il est difficile de mettre en œuvre des programmes préventifs et curatifs pour l'amélioration de la santé des enfants comme celle des adultes. Il en va de même des programmes visant la lutte contre le paludisme. Il est évident que la prévalence du paludisme est élevée dans les zones à conflits comme le Kasaï.

Le coût élevé des soins de santé dans les formations sanitaires par rapport au revenu de la population constitue un défi majeur. Les ménages constituent la principale source et un acteur clé du financement de la santé en RDC dans une proportion de $41 \%$ des dépenses courantes de santé (Makamba et Manunga, 2004). La dépense courante des ménages est en grande partie affectée à des dépenses pour les soins curatifs $(64 \%)$, pour l'achat des médicaments délivrés sur ordonnance (30\%) et pour les soins préventifs $2 \%$ (MSP, 2016). La prévention du paludisme en RDC est essentiellement orientée vers la lutte anti-vectorielle. Il s'agit de l'utilisation de la moustiquaire imprégnée d'insecticide, promotion de l'assainissement et la pulvérisation intra domiciliaire. L'utilisation de la moustiquaire imprégnée d'insecticide à grande échelle est une stratégie adoptée par le PNLP dans la prévention du paludisme chez les enfants de moins de cinq ans (PNLP-RDC, 2017). La lutte contre le parasite est essentiellement tournée vers le traitement préventif intermittent au cours de la grossesse pour les femmes enceintes(Losimba et al., 2018).

Les données de cette étude proviennent de l'Enquête Démographique et de Santé (EDS-RDC II) réalisé entre 2013 et 204 en RDC. L'opération a été 
réalisée sur le terrain entre fin novembre 2013 et début février 2014. Les vingtsix nouvelles provinces ont constitué les domaines d'études. Dans chaque domaine d'étude, trois strates ont été créées : la strate des villes statutaires, la strate des cités et la strate du milieu rural. Le tirage de l'échantillon a été fait strate par strate. L'EDS-RDC II s'est basé sur un sondage aréolaire, stratifié au niveau des unités primaires et tiré à plusieurs degrés. Pour les strates des villes statutaires et des cités, au premier degré, un certain nombre de quartiers, à partir de la liste exhaustive de l'ensemble des quartiers des villes ou des cités, a été tiré. Au second degré, on a tiré 34 ménages dans les quartiers sélectionnés au premier degré.

Pour les strates du milieu rural, on a tiré au premier degré le secteur ou la chefferie et au deuxième degré un ou plusieurs villages dans les secteurs et chefferies tirés au premier degré. Au troisième degré, on a tiré 34 ménages dans les villages tirés au deuxième degré. Au-delà 500 ménages dans la grappe, le quartier ou le village échantillonné a été subdivisé en plusieurs segments parmi lesquels un seul a été retenu dans l'échantillon. À l'opposé, les villages de petite taille ont été regroupés.

Au premier degré, le tirage a été effectué avec probabilité proportionnelle à la taille en population des unités de sondage. Au total, 540 grappes (quartier ou village) ont été tirées. Par contre, aux deuxième et troisième degrés, on a procédé à un tirage systématique avec probabilité égale. Au total de 18360 ménages (5 474 en milieu urbain dans 161 grappes et 12 886 en milieu rural dans 379 grappes) ont été tirés.

La base de sondage de l'EDS RDC II est le recensement scientifique de la population de 1984. Elle a été actualisée partiellement à plusieurs reprises par des recensements administratifs et récemment dans le cadre des élections présidentielles et législatives de 2011. Cette base de sondage est la même que celle qui a été utilisée par l'enquête Multiple Indicator Cluster Surveys (MICS) de 2010 et par l'enquête 1-2-3 (Emploi, Secteur Informel et Consommation des ménages) de 2012, menées par l'Institut National de la Statistique (INS).

Toutes les femmes âgées de 15-49 ans vivantes habituellement dans les ménages sélectionnés, ou présentes la nuit précédant l'enquête, étaient éligibles pour être enquêtées. De plus, dans un sous-échantillon d'un ménage sur deux, tous les hommes de 15-59 ans étaient éligibles pour être enquêtés. Dans ce sous-échantillon, 8489 enfants de 6-59 mois étaient également éligibles pour un test du paludisme et pour des tests de couverture vaccinale.

\section{Variables}

Deux tests ont permis le dépistage du paludisme. Le Test de Diagnostic Rapide (TDR), plus précisément le SD BIOLINE Malaria Antigen P.f, et la goutte épaisse. Le TDR identifie les antigènes et non parasites. La mesure des 
antigènes au lieu des parasites se traduit par le fait qu'un test sera positif pendant un certain temps après le traitement réussi d'une infection en raison des antigènes résiduels. De ce fait, il donne un nombre élevé de cas que la goutte épaisse.

La goutte de sang était obtenue à partir de la même piqûre au bout du doigt effectuée pour le test d'hémoglobine et le TDR. Après séchage, les lames étaient rangées dans des boîtes spéciales avec des dessicatifs et des contrôleurs d'humidité. Ces boîtes étaient transférées de façon régulière au Laboratoire National de Référence (LNR) pour la recherche d'hématozoaires par microscopie. Le résultat de la microscopie constitue la variable dépendante de cette étude. Cette variable est une variable dichotomique, elle distingue les enfants de 6-59 mois testés positifs au paludisme de ceux qui n'ont pas été positifs.

Les variables indépendantes font références aux caractéristiques de la communauté (province de résidence, milieu de résidence et proportion des pauvres), caractéristiques du ménage (le niveau de vie, promiscuité dans le ménage, la disponibilité de la moustiquaire dans le ménage et type de toilettes), les caractéristiques de la mère et de l'enfant (ethnie de la mère, âge de la mère, religion de la mère, niveau d'instruction de la mère, occupation de la mère, culture de la mère sur le paludisme utilisation de la moustiquaire, sexe de l'enfant et âge de l'enfant). La variable niveau de vie est créé à partir du confort de l'habitat et possessions de certains biens par le ménage (Cf. annexe 2). Une analyse en composantes principales a été ensuite effectuée. La première composante a enfin été retenue comme indicateur de niveau de vie. La variable culture de la mère sur le paludisme a été construite à partir de 17 variables. De ces variables, 6 sur les modes de transmission, 7 sur les symptômes de la maladie et 4 sur les modes de prévention (Cf. Annexe 3).

\section{Méthodes d'analyse}

$\mathrm{Ce}$ travail utilise, en premier lieu, l'Analyse Factorielle des Correspondances Multiples (AFCM). Cette étude caractérise d'abord les principaux axes factoriels et ensuite, utilise le plan factoriel pour affiner notre typologie afin de dégager le profil des enfants selon leur état paludique. La caractérisation des principaux axes est faite sous SPSS 25 et le plan factoriel sous SPAD 5.5. Ainsi, en second lieu, cette étude fait recours à la régression logistique multiniveau à effets aléatoires. La modélisation avec les effets aléatoires se fait en considérant deux hypothèses (Givord et Guillerm, 2016). La première est que la distribution des effets groupes inobservés suit une loi normale (ou tout au moins une loi paramétrique qu'on peut spécifier). La seconde est que ces effets groupes sont indépendants des variables explicatives. Cette deuxième hypothèse est forte et difficile à être vérifiée. 
Formellement, l'équation s'écrit :

$$
Y_{i j}=\beta_{0}+\beta x_{i j}+\gamma x_{j}+\alpha_{j}+\varepsilon_{i j} \quad j=1, \ldots, J \quad i=1, \ldots, n_{j}
$$

Sous les hypothèses : $\varepsilon_{i j} \sim N\left(0, \sigma_{\varepsilon}^{2}\right)$ et $\alpha_{j} \sim N\left(0, \sigma_{\alpha}^{2}\right)$

Les paramètres $\beta_{0}, \beta$ et $\gamma$ correspondent respectivement à la constante, au vecteur des variables explicatives de niveau 1 et au vecteur des variables explicatives de niveau 2. $\sigma_{\varepsilon}^{2}$ et $\sigma_{\alpha}^{2}$ représentent respectivement les variances intra-groupe et inter-groupe. Ces variances permettent de calculer l'ICC (Intra Class Correlation).

$$
\Omega=\frac{\sigma_{\alpha}^{2}}{\left(\sigma_{\alpha}^{2}+\sigma_{\varepsilon}^{2}\right)}
$$

L'ICC $(\Omega)$ correspond à la part de la variance expliquée par l'effet groupe et peut être utilisé comme un premier indicateur de l'utilité de recourir à une modélisation multiniveau (Koné, 2012). Lorsque $\Omega$ est proche de 1 , cela signifie que les unités au sein d'un même groupe sont très proches et se distinguent beaucoup de celles des autres groupes. A l'inverse, si $\Omega$ est proche de zéro, cela signifie que les observations à l'intérieur d'un même groupe ne sont pas plus semblables entre elles que des observations de groupes différents.

Quatre modèles sont estimés dans ce travail : M0, M1, M2 et M3. Le modèle M0 ou modèle vide fournit la répartition de la variance entre les différents niveaux, grâce aux termes aléatoires inclus à chacun d'eux. Les modèles M1 et M2 rendent compte de la variabilité de la variance entre les communautés par rapport au modèle vide M0. Dans le modèle M3 (modèle complet), les variables explicatives des niveaux 1 et 2 sont toutes introduites dans le modèle vide. Ce modèle complet permet de distinguer l'effet des caractéristiques individuelles de l'effet des caractéristiques de la communauté, ainsi que les effets aléatoires propres à chaque niveau (Nganawara, 2016).

La qualité de l'ajustement des modèles est faite à partir du test de Wald au seuil de 5\%. Le test de Wald dans les modèles multiniveaux permet de comparer un modèle courant au même modèle multiniveau mais ayant uniquement la constante dans la partie fixe. Il permet de rejeter l'hypothèse selon laquelle le vecteur des effets des différents coefficients est nul et de conclure qu'au moins une variable indépendante exerce une influence sur la variable dépendante et donc de justifier la significativité du modèle. Les valeurs des coefficients d'un modèle multiniveaux binaire sont à interpréter avec précaution car leur valeur dépend directement des choix de modélisation et notamment de la contrainte identifiante qui consiste à fixer la variance des résidus individuels (Givord et Guillerm, 2016). Elle vaut classiquement $\frac{\pi^{2}}{3}$ dans le modèle logit (variance d'une loi logit standard) et 1 dans le modèle probit. 


\section{Présentation des résultats}

\section{Analyse descriptive multivariée de l'état palustre chez les enfants de 6-59} mois

\section{Interprétation des axes}

L'objectif des analyses factorielles est d'étudier le profil des enfants impaludés en RDC. Les trois premiers axes factoriels (1,2 et 3 ) contribuent respectivement à $14,58 \%, 9,72 \%$ et $6,6 \%$, soit environ $30,9 \%$ de 1 'inertie totale. L'axe 1 ordonne les enfants selon le milieu de résidence, la proportion des ménages pauvres dans la communauté, le niveau de vie du ménage, le niveau d'instruction et l'occupation de la mère (respectivement 17,15\%, $15,59 \%, 20,88 \%, 11,09 \%$ et $8,77 \%$ ). L'axe 2 est constitué principalement de la disponibilité des moustiquaires dans le ménage et de l'utilisation de la moustiquaire (respectivement 14,96\% et 15,31\%). L'axe 3 organise les enfants selon la province de résidence, l'ethnie et la religion de la mère (respectivement $33,94 \%, 32,58 \%$ et 8,27\%). Ainsi, l'axe 1 peut être interprété comme l'axe socio-économique, l'axe 2 comme celui de la disponibilité des moyens préventifs dans le ménage et du comportement préventif de la mère et l'axe 3 est interprété comme l'axe culturel.

\section{Profil des enfants par rapport au paludisme}

Le Graphique 1 illustre le premier plan factoriel (axes 1 et 2) dans lequel deux grands groupes d'enfants s'opposent diamétralement. Dans le premier groupe à gauche se retrouvent les enfants impaludés, qui résident en milieu rural et dans les provinces du Centre, du Nord-est et du Nord-ouest. En général, ces enfants vivent dans les communautés où la proportion des pauvres est élevée. Ils appartiennent à des ménages pauvres qui ne disposent d'aucune moustiquaire, de ce fait, ils ne dorment pas sous une moustiquaire. Leurs mères sont agricultrices et sans niveau d'instruction ou de niveau primaire. Le deuxième groupe regorge d'enfants non impaludés, qui vivent en milieu urbain et dans les provinces de l'Ouest et du Sud-est. Dans l'ensemble, ces enfants appartiennent à des communautés à faible proportion des ménages pauvres. Ils sont issus des ménages de niveau de vie moyen ou élevé qui possèdent une moustiquaire pour deux personnes ou pour plus de deux personnes. De ce fait, ils dorment davantage sous une moustiquaire. Leurs mères sont actives non agricultrices ou inactives et de niveau d'instruction secondaire ou plus. 
Graphique 1. Profil des enfants par rapport au paludisme

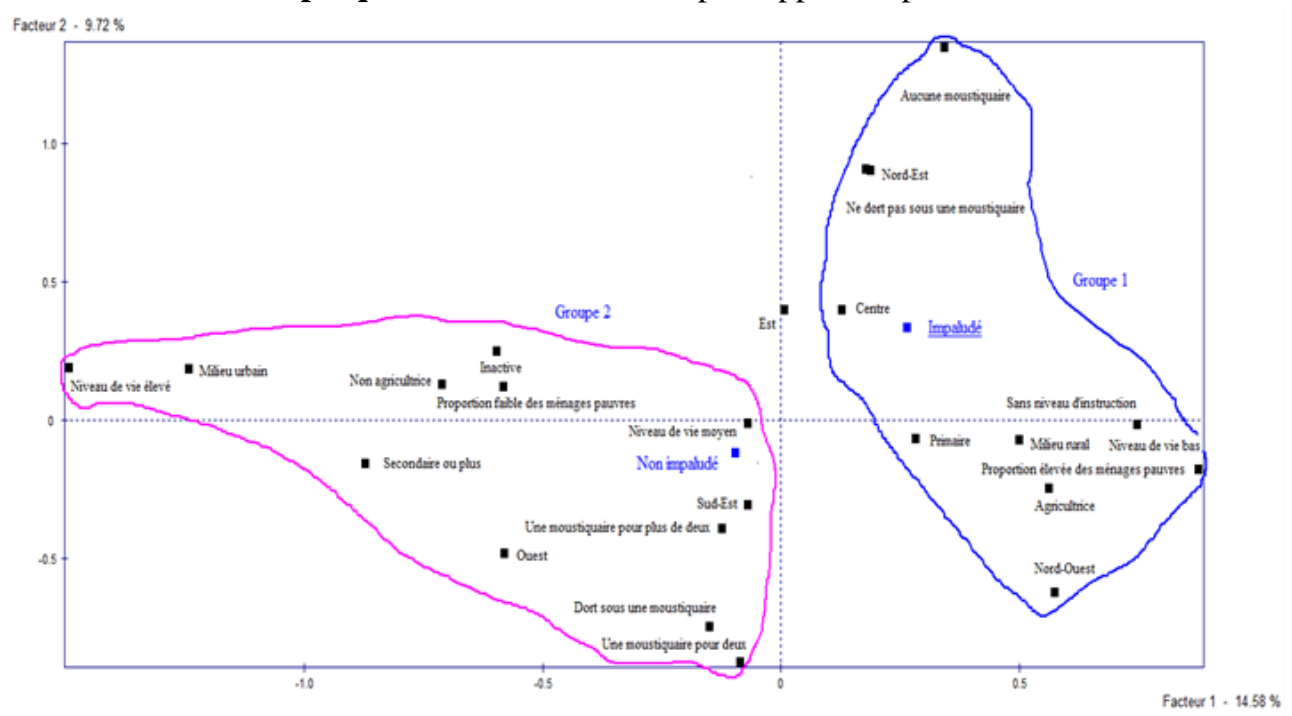

Source : Exploitation données de l'EDS-RDC II, 2013-2014

\section{Présentation du résultat de la régression multiniveau}

La mise en évidence de la décomposition de la variance montre que les caractéristiques propres aux communautés influencent la survenue du paludisme chez l'enfant. Le modèle M0 (tableau 1) montre que la part de la variance attribuable à la communauté est de $41,88 \%$ dans l'explication du risque de contracter le paludisme chez les enfants de 6-59 mois. L'effet des caractéristiques individuelles (caractéristiques du ménage, de la mère et de l'enfant) sur le risque de contracter le paludisme (tableau 1 ; modèle M1). Il en ressort que les caractéristiques individuelles ont un impact sur la survenue du paludisme chez l'enfant, car elles permettent d'améliorer le modèle nul. L'introduction des variables individuelles au modèle nul baisse la variance entre les communautés d'environ 11,67\% (Tableau 1, Modèle 1). Ce modèle explique significativement la survenue du paludisme chez les enfants $\left(X^{2}=248,87(25 \mathrm{dl}), \mathrm{p}<0,000\right)$.

Les caractéristiques communautaires introduites dans le modèle nul apportent également une contribution dans l'explication du paludisme chez les enfants (Tableau 1, modèle M2). Ces caractéristiques de la communauté influencent de façon favorable ou défavorable le risque d'accès palustre des enfants. Ces effets sont significatifs à l'exception de la variable proportion des pauvres au sein d'une communauté. En référence au modèle nul, la variance entre les communautés diminue de de 9,31\% (Tableau 1, Modèle M2). Ce modèle explique significativement le risque de contracter le paludisme chez les enfants $\left(X^{2}=65,91(7 \mathrm{dl}), \mathrm{p}<0,000\right)$. 
Le modèle complet inclut les effets des caractéristiques individuelles et ceux des caractéristiques de la communauté dans l'explication de la survenue de l'infection palustre chez les enfants (Tableau 1, Modèle M3). La diminution de la variance entre les communautés par rapport au modèle nul est plus importante que celle que l'on observe dans les modèles M1 et M2, soit 13,38\% (Tableau 1, Modèle M3). L'introduction des variables explicatives individuelles et contextuelles dans le modèle nul contribue donc à mieux appréhender les facteurs influençant la survenue du paludisme chez les enfants. Ce modèle explique significativement le risque de contracter le paludisme chez les enfants de 6-59 mois $\left(\mathrm{X}^{2}=258,87\right.$ (32dl), $\left.\mathrm{p}<0,000\right)$. La prise en compte de toutes les variables dans le modèle apporte quelques changements dans l'influence de chacune des caractéristiques retenues. Par rapport au modèle $\mathrm{M} 1$, l'ethnie de la mère devient non significative et le type de lieu d'aisance significatif. La significativité du niveau de vie des ménages s'améliore. Par rapport au modèle M2, le milieu de résidence devient non significatif et la significativité de la province de résidence se détériore. Dans ce modèle M3, le risque de contracter le paludisme augmente avec l'âge de l'enfant. Les enfants de 24-59 mois ont 2,14 fois plus de risques de contracter le paludisme que ceux moins âgés de 6-23 mois. L'utilisation de la moustiquaire baisse significativement le risque de contracter le paludisme : les enfants qui ne dorment pas sous une moustiquaire ont 1,24 fois plus de risques de contracter le paludisme.

Le risque de contracter le paludisme chez les enfants baisse avec le niveau d'instruction de la mère. Comparativement aux enfants des mères sans niveau d'instruction, les enfants des mères de niveau secondaire ou plus ont $39,2 \%$ moins de risques de contracter le paludisme. Le risque de contracter le paludisme diminue significativement avec l'augmentation du niveau de vie. Les enfants des ménages de niveau de vie moyen et élevé ont respectivement $13,8 \%$ et $45 \%$ moins de risques de contracter le paludisme. La promiscuité influence significativement la survenue du paludisme chez les enfants. Les enfants des ménages dont une pièce est partagée par plus de trois personnes ont 1,18 fois plus de risques de contracter le paludisme que leurs homologues des ménages dont une pièce est partagée par 1-3 personnes.

$\mathrm{La}$ province de résidence influence fortement la survenue du paludisme chez les enfants de 6-59 mois. Les enfants des provinces du centre et ceux du Sud-est ont respectivement 2,54 et 2,93 fois plus de risques de contracter le paludisme que leurs homologues qui résident dans les provinces de l'Ouest.

La proportion des pauvres dans la communauté, le milieu de résidence, la disponibilité des moustiquaires, la culture de la mère sur le paludisme, l'occupation, la religion et l'ethnie de la mère et le sexe de l'enfant ne discriminent pas significativement les enfants face au paludisme. 
Tableau 1. Déterminants individuels et contextuels du paludisme chez l'enfant

\begin{tabular}{|c|c|c|c|c|c|}
\hline Variables & Modalités & M0 & M1 & M2 & M3 \\
\hline Partie fixe & Constante & $0,233^{* * * *}$ & $0,253^{* * *}$ & $0,157^{* * *}$ & $0,122^{* * * *}$ \\
\hline \multicolumn{6}{|c|}{ Caractéristiques individuelles } \\
\hline \multirow{2}{*}{ Sexe de l'enfant } & Masculin & & Réf & & Réf \\
\hline & Féminin & & 1,040 & & 1,043 \\
\hline \multirow{2}{*}{ Age de l'enfant } & 6-23 mois & & Réf & & Réf \\
\hline & $24-59$ mois & & $2,134^{* * *}$ & & $2,142^{* * * *}$ \\
\hline \multirow{2}{*}{$\begin{array}{l}\text { Utilisation de la } \\
\text { moustiquaire }\end{array}$} & Utilise & & Réf & & Réf \\
\hline & N'utilise pas & & $1,255^{* *}$ & & $1,241^{* * *}$ \\
\hline \multirow[t]{7}{*}{ Ethnie de la mère } & Kasaï-Katanga et Tanganyika & & Réf & & Réf \\
\hline & Bakongo & & 0,724 & & 1,599 \\
\hline & Bas Kasaï_Kwilu Kwango & & $0,415^{* * *}$ & & 0,812 \\
\hline & Cuvette Centrale & & $0,425^{* * *}$ & & 0,914 \\
\hline & Ubangi et Itimbiri & & $0,637^{* *}$ & & 1,914 \\
\hline & Uele_Lac_Albert & & 1,179 & & 1,822 \\
\hline & Basele Komo maniema et kivu & & $0,384^{* * *}$ & & 1,043 \\
\hline \multirow[t]{3}{*}{ Religion de la mère } & Catholique & & Réf & & Réf \\
\hline & Protestante & & 0,959 & & 0,949 \\
\hline & Autre religion & & 0,918 & & 0,926 \\
\hline \multirow[t]{3}{*}{ Age de la mère } & $15-24$ ans & & Réf & & Réf \\
\hline & $25-34$ ans & & 1,055 & & 1,056 \\
\hline & $35-49$ ans & & 1,104 & & 1,111 \\
\hline \multirow{3}{*}{$\begin{array}{l}\text { Niveau d'instruction } \\
\text { de la mère }\end{array}$} & Sans niveau & & Réf & & Réf \\
\hline & Primaire & & 0,964 & & 1,949 \\
\hline & Secondaire ou plus & & $0,633^{* * * *}$ & & $0,608^{* * * *}$ \\
\hline \multirow{3}{*}{$\begin{array}{l}\text { Occupation de la } \\
\text { mère }\end{array}$} & Agricultrice & & Réf & & Réf \\
\hline & Inactive & & 0,998 & & 0,980 \\
\hline & Non agricultrice & & 0,941 & & 0,912 \\
\hline \multirow{3}{*}{$\begin{array}{ll}\text { Culture sur } & \text { le } \\
\text { paludisme } & \end{array}$} & Faible & & Réf & & Réf \\
\hline & Moyenne & & 0,939 & & 0,946 \\
\hline & Forte & & 0,907 & & 0,932 \\
\hline \multirow{3}{*}{$\begin{array}{l}\text { Niveau de vie du } \\
\text { ménage }\end{array}$} & Bas & & Réf & & Réf \\
\hline & Moyen & & 0,888 & & $0,862^{*}$ \\
\hline & Elevé & & $0,583^{* * *}$ & & $0,550^{* * * *}$ \\
\hline \multirow[t]{2}{*}{ Promiscuité } & 1-3 Personne par pièce & & Réf & & Réf \\
\hline & Plus 3 personne par pièce & & $1,174^{* *}$ & & $1,177^{* * *}$ \\
\hline \multirow{2}{*}{$\begin{array}{l}\text { Type de lieu } \\
\text { d'aisance }\end{array}$} & Toilette non améliorée & & Réf & & Réf \\
\hline & Toilette améliorée & & 0,848 & & $0,848^{*}$ \\
\hline \multirow{3}{*}{$\begin{array}{l}\text { Disponibilité } \quad \mathrm{de} \\
\text { moustiquaire }\end{array}$} & Aucune moustiquaire & & Réf & & Réf \\
\hline & $\begin{array}{l}\text { Une moustiquaire pour plus de } \\
\text { deux }\end{array}$ & & 0,981 & & 0,985 \\
\hline & Une moustiquaire pour deux & & 0,958 & & 0,970 \\
\hline \multicolumn{6}{|c|}{ Caractéristiques communautaires } \\
\hline \multirow[t]{4}{*}{ Provinces } & Ouest & & & Réf & Réf \\
\hline & Nord-ouest & & & 1,451 & 0,947 \\
\hline & Centre & & & $2,992^{* * *}$ & $2,536^{* * *}$ \\
\hline & Sud-est & & & $3,356^{* * *}$ & $2,933^{* * * *}$ \\
\hline
\end{tabular}




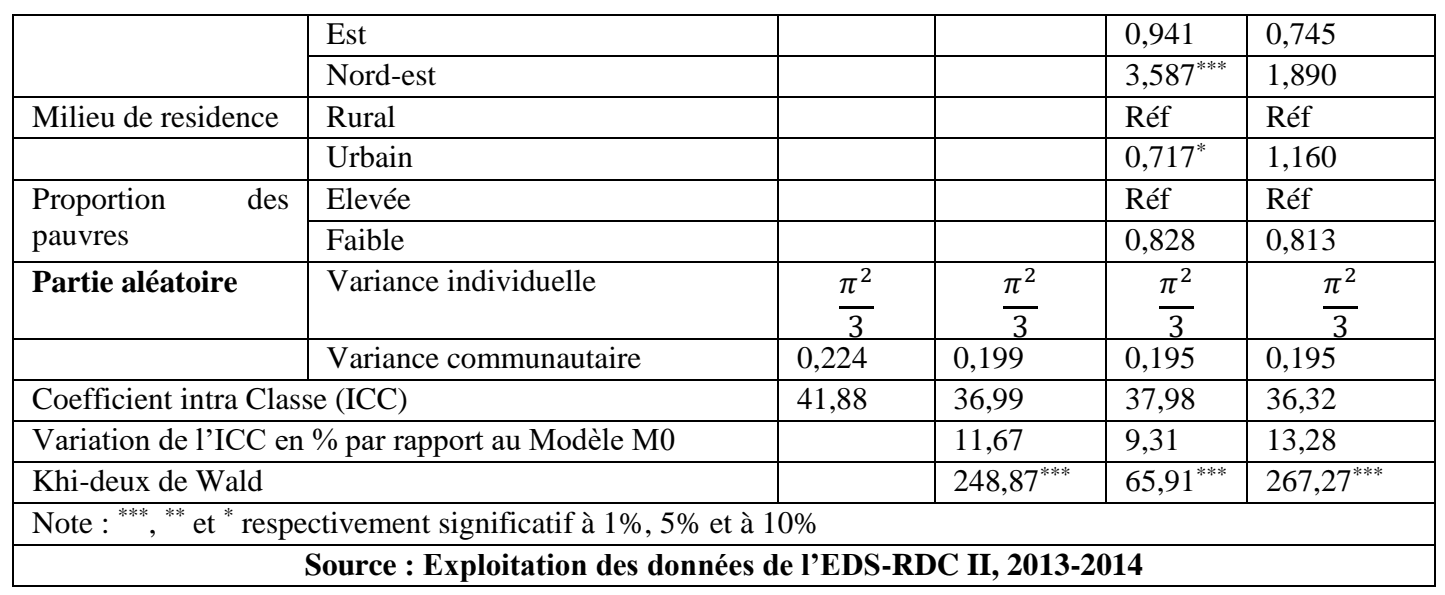

\section{Discussion et Conclusion}

Les résultats montrent que les enfants des provinces du centre et ceux du Sud-est ont plus de risques de contracter le paludisme que leurs homologues qui résident dans les provinces de l'Ouest. Ces résultats révèlent une disparité régionale face à la survenue du paludisme chez les enfants en RDC. Ces résultats corroborent ceux trouvés dans les études antérieures au Niger (Abdou, 2014) ; en Guinée (Barry, 2015) ; au Mali (Diallo, 2016) ; au Bénin (Samakou, 2017) ; au Burkina Faso (Kaboré, 2019 ; Ouedragaogo et al., 2018). Les caractéristiques climatiques, entomologiques et parasitologiques confèrent à chacune des provinces du centre et du Sud-est un faciès (Remy, 1988 ; Maud, 2006). Les provinces du Centre sont caractérisées par un climat tropical humide dans l'ex-Kasaï oriental (Sankuru, Lomami et Kasaï oriental) et un climat tropical équatorial dans l'ex-Kasaï occidental (Kasaï et Kasaï central) (PNUD, 2009b ; PNUD, 2009c). La saison des pluies dure huit mois et la saison sèche quatre mois. Dans l'ex-Kasaï oriental, la saison des pluies s'étend de septembre à avril et la saison sèche de mai à août (PNUD, 2009c). Dans l'ex-Kasaï occidental, la saison des pluies va de mi-août à mi-janvier et de mi-février à mi-mai et la saison sèche quant à elle va de mi-janvier à mifévrier et de mi-mai à mi-août (PNUD, 2009b). Les provinces du Sud-est ou l'ex-Katanga (Tanganyika, Haut-Lomami, Haut-Katanga et Lualaba) connaissent un climat tropical humide et un climat tempéré (PNUD, 2009d). Les conditions climatiques de ces provinces favorisent la prolifération des moustiques. La fréquence des pluies explique l'abondance des anophèles (Doumbia, 2007). Les pluies abondantes sont par ailleurs dévastatrices des gîtes larvaires (Capelle, 2007 ; Pierrat, 2010). Ces provinces du centre et du Sud-est font partie du faciès tropical avec au moins 60 à 400 piqûres infectées par personne et par an. La prémunition apparaît plus tardivement, vers l'âge de 10 ans (MPSMRM, MSP et ICF International, 2014). Les conditions socio- 
économiques et politiques des provinces du centre et du Sud-est renforcent cette vulnérabilité des enfants face au paludisme (UNICEF, 2018).

Le Gouvernement a besoin de ressources pour prévenir la survenue du paludisme chez les enfants (Itriago, 2011). L'assiette restreinte des recettes fiscales ne permet pas aux pouvoirs publics de la RDC de mobiliser les revenus nécessaires pour financer leurs propres activités et fournir des services publics (Banque Mondiale, 2018). La part du budget de l'Etat consacrée à la santé est modique et largement inférieure aux engagements d'Abuja (15\%) et l'aide extérieure insuffisante (MSP, 2016; BCC, 2018). Par ricochet, la part du budget de la santé allouée aux activités de la lutte antipaludique reste faible dans tout le pays (PNLP-RDC, 2017). De ce fait, les activités et les zones d'interventions du PNLP deviennent de plus en plus sélectives. Les grandes campagnes de distribution des moustiquaires se font plus dans les provinces de l'Ouest que dans les provinces du Centre et du Sud-est (Losimba et al., 2018). Les ménages des provinces du centre et du Sud-est possèdent moins de moustiquaires que ceux de l'Ouest (INS-RDC, 2018). La possession des moustiquaires dans le ménage n'est pas synonyme de l'accès à la moustiquaire et de son utilisation (USAID, 2014). Néanmoins, cette augmentation en possession de moustiquaires imprégnées d'insecticides a permis d'améliorer l'accès de la population aux moustiquaires et même le taux d'utilisation, aussi bien en milieu urbain qu'en milieu rural (Losimba et al., 2018).

Les provinces de l'Ouest connaissent de moins en moins les situations d'instabilités, les guerres et autres, contrairement aux provinces du Centre et du Sud-est. Les affrontements entre les milices de Kamuina Nsapu et les forces de défense et de sécurité dans les provinces du centre sont susceptibles d'empêcher toute activité préventive contre le paludisme ${ }^{6}$. Pendant ces violences, certaines familles ont vécu avec leurs enfants à ciel ouvert sans accès à l'eau salubre, aux soins de santé ni à la moindre moustiquaire pour se protéger de la malaria (UNICEF, 2018). L'instabilité politique et les violences freinent et empêchent la mise en œuvre des programmes de développement (Barbieri, 1991).

Les résultats montrent que le risque de contracter le paludisme diminue avec l'amélioration du niveau de vie des ménages. Longuepée (2006); Yovoga (2009) ; Nkemba et al. (2014) ; Barry (2015) ; Fournet et al. (2015) ;

${ }^{6}$ L'insurrection des Kamuina Nsapu a été déclenchée en août 2016, lorsque le chef coutumier du même nom (Jean-Pierre Mpandi) a été exécuté avec plusieurs de ses adeptes au cours d'un assaut mené à son domicile par les forces de défense et de sécurité congolaises. Jean-Pierre Mpandi était entré en confrontation ouverte avec les autorités nationales depuis que celles-ci avaient refusé de reconnaître officiellement son statut de chef coutumier Kamuina Nsapu, un droit pourtant consacré en vertu de la loi de 2015 fixant le statut des chefs coutumiers. De mars à juillet 2017, il y a eu d'exécutions sommaires, des actes de torture, des mutilations, des violences sexuelles, des pillages, des destructions de biens, des arrestations et des détentions arbitraires et des transferts forcés de populations. 
Saïdou (2018) ; Ouedragaogo et al. (2018) et Kaboré (2019) ont abouti à des résultats similaires. En dépit des campagnes de distribution gratuite de moustiquaires, les moyens préventifs (la moustiquaire, l'insecticide et le spirale anti-moustique etc.) ont un coût (Takudzwa, 2018). Les coûts de prévention du paludisme en Afrique sont en fait assumés par la population (Nkemba et al., 2014). Il existe une relation étroite entre l'économie des ménages et le paludisme (Longuepée, 2006). La structure des dépenses des ménages pauvres en RDC révèle une prédominance des dépenses incompressibles (aliment, logement, etc.) (PNUD, 2009a). Dans ces ménages, l'arbitrage entre l'achat des produits préventifs contre le paludisme et les besoins incompressibles reste en faveur des besoins incompressibles. Ces ménages réduisent les fonds alimentaires pour faire face aux dépenses médicamenteuses (Diallo, 2003). Les femmes chef de ménage sont contraintes de sacrifier leur jour de travail pour s'occuper des enfants atteints de paludisme (Pierrat, 2010).

La qualité du logement et la nature de sa construction, sa conception, les matériaux utilisés et l'emplacement sont considérés comme ayant un impact sur la vulnérabilité des résidents du ménage au paludisme (Takudzwa, 2018). Les enfants des ménages de niveau de vie moyen ou élevé vivent dans des conditions d'habitations moins favorables aux moustiques (Kaboré, 2019). L'amélioration des logements diminue le risque de contracter le paludisme en raison de la diminution de l'entrée des moustiquaires dans la maison moderne. Les maisons équipées d'écrans ou d'avant toits fermés ont un effet protecteur sur les occupants contre la menace des piqûres des moustiques infectés. Toutefois, les enfants de ménages riches contractent également le paludisme. Ces ménages riches possèdent de l'électricité ou des générateurs (Pierrat, 2010). La lumière électrique attire les vecteurs du paludisme, d'où son utilisation comme pièges à moustiques (Takudzwa, 2018). Cependant, le lien entre le paludisme et l'électricité reste complexe et exige des études pour une compréhension plus poussée (Takudzwa, 2018). En outre, les ménages riches ont tendance à vivre en ville et repoussent les animaux domestiques. D'où, la concentration des piqûres sur l'homme qui est la seule proie disponible pour l'anophèle (Remy, 1988 ; Pierrat, 2010).

Les résultats montrent que la promiscuité augmente le risque de contracter le paludisme chez les enfants (Fournier et Haddad, 1995 ; Maud, 2006 ; Pierrat, 2010; Takudzwa, 2018). En RDC, le développement économique ne suit pas la croissance de la population. Ceci provoque une concentration de citadins très pauvres dans des quartiers dégradés, ou, plus généralement, dans des zones périphériques sous-équipées. Dans ces quartiers, les ordures ménagères traînent partout et l'eau stagne dans les cours à l'hivernage provoquant ainsi le développement des moustiques (PNUD, 2009a). A Kinshasa, comme dans les autres villes du pays, les pauvres sont 
entassés et plusieurs ménages partagent souvent une même concession. La taille moyenne des ménages est plus élevée dans les centres urbains (MPSMRM, MSP et ICF International, 2014). Les résultats montrent que les enfants des ménages qui utilisent les toilettes améliorées contractent moins le paludisme que les enfants des ménages qui utilisent les toilettes non améliorées. Kaboré (2019) est arrivé à la même conclusion. Les eaux des toilettes non améliorées stagnent parfois dans la cours ou dans un trou creusé par les membres du ménage. Les anophèles ont une forte capacité d'adaptation (Capelle, 2007). Ces eaux usées qui stagnent deviennent, de ce fait, le lieu de développement des moustiques.

Les enfants des mères de niveau d'instruction secondaire ou plus ont moins de risque d'attraper le paludisme par rapport aux enfants des mères de niveau primaire ou sans niveau. Ces résultats concordent avec ceux trouvés par Pierrat (2010) ; Fournet et al. (2015) ; Diallo (2016) et Kaboré (2019). En RDC, peu de femmes ont un niveau d'instruction secondaire ou plus (MPSMRM, MSP et ICF International, 2014). De tels constats montrent que le niveau d'instruction élevée distingue une femme congolaise dans la société et dans son foyer. Ce niveau élevé d'instruction confère à la femme un statut public et un statut privé (Rakotondrabe, 2004) et la fait participer à la prise de décision dans le couple en matière de santé et d'hygiène des enfants. Le niveau d'instruction élevée atténue le poids de la tradition et des pratiques défavorables à la santé des enfants (Franckel, 2004). Le niveau d'instruction de la mère a une influence sur le comportement sanitaire du ménage (Rakotondrabe, 2004). Les mères instruites sont favorables et assimilent correctement les conseils préventifs sur le paludisme (Pierrat, 2010). L'instruction de la mère influence les connaissances, attitudes et pratiques du ménage au sujet de la morbidité palustre (Takudzwa, 2018).

En outre le niveau d'instruction de la femme congolaise est associé à son statut socio-économique. Les femmes de niveau d'instruction supérieure ou plus font partie de la catégorie de femmes de niveau d'instruction secondaire ou plus. Ces femmes de niveau d'instruction supérieure ou plus trouvent davantage du travail que leurs homologues hommes. Le taux de chômage des femmes est inférieur à celui des hommes (INS-RDC, 2014). Il est susceptible que ces femmes accèdent aux revenus plus élevés qui pourraient aussi leur permettre de se procurer des moyens préventifs contre le paludisme. Les enfants des mères instruites vivent dans des ménages de niveau de vie élevé ayant diverses possibilités de protéger contre les piqûres des moustiques. Outre la possibilité de se procurer des insecticides, des spirales anti-moustiques, ces enfants des mères instruites vivent dans les milieux urbanisés qui repoussent les gîtes larvaires des moustiques (Maud, 2006).

Les résultats montrent que les enfants qui ne dorment pas sous une moustiquaire sont davantage impaludés. Ces résultats corroborent ceux 
trouvés Au Malawi (Hajison et al., 2018); Au Burkina Faso (Bouba Djourdebbé et al., 2018) ; Au Zimbabwe (Takudzwa, 2018). Toutefois, les enfants qui dorment sous une moustiquaire contractent aussi le paludisme (Kaboré, 2019). Ceci s'explique par le fait que l'anophèle est aussi en activité avant $23 \mathrm{~h}$ quoi qu'elle soit réputée être en activité maximale entre $23 \mathrm{~h}$ et $6 \mathrm{~h}$ (Audry et Gaüzère, 2019). Dans les zones périphériques des milieux urbains où vivent les pauvres, les enfants sont contraints d'attendre la cuisson des aliments jusqu'aux heures tardives (Takudzwa, 2018). Parfois, ces enfants jouent également jusqu'à des heures tardives.

Chez les enfants de 6-59 mois, le risque d'attraper le paludisme augmente avec l'âge de l'enfant. Sabatinelli et al. (1986), Othingue (2005), Mutombo et al. (2018), Saïdou (2018) et Kaboré (2019) ont abouti aux résultats similaires. Ce résultat s'explique par le fait que certains groupes d'âges sont plus vulnérables que d'autres (Takudzwa, 2018). Les enfants de moins d'un an sont moins vulnérables au paludisme en raison des anticorps acquis dans l'utérus de leur mère (Carnevale et Vaugelade, 1987 ; Takudzwa, 2018). Il a été constaté que les garçons plus sensibles que les filles (Le Hersan, 2000). En outre, les enfants de 6-23 mois bénéficient de plus de protection et d'attention que leurs ainés de 24-59 mois (Pierrat, 2010). Généralement, les mères ont tendance à leur faire porter les habits longs qui laissent de moins en moins la peau du corps visible (ANOFEL, 2014).

La province de résidence, le niveau de vie des ménages, le type de lieu d'aisance, la promiscuité dans le ménage, le niveau d'instruction de la mère, l'utilisation de la moustiquaire et l'âge de l'enfant déterminent significativement le paludisme chez les enfants de 6-59 mois. Ainsi, ces hypothèses spécifiques associées à ce travail ont été vérifiées. La moustiquaire réduit le risque de contracter le paludisme. Le risque de contracter le paludisme diminue avec le niveau de vie et le niveau d'instruction de la mère. La province de résidence influence la survenue du paludisme chez les enfants de 6-59 mois.

Toutefois, ce travail a des limites liées aux données et à la méthodologie utilisée qu'il convient de signaler. Les variables composites facilitent certes l'interprétation, cependant elles ont le défaut d'être subjectives. Egalement, la zone de dénombrement utilisée pour définir les communautés n'est pas une entité. Dans l'attente d'un potentiel vaccin, pour atteindre les objectifs d'élimination du paludisme ici de 2030, nous formulons les recommandations ci-après au gouvernement et aux partenaires au développement : de mener des actions préventives dans les provinces du Centre (Sankuru, Lomami, Kasaï oriental, Kasaï et Kasaï central et du Sudest (Tanganyika, Haut-Lomami, Haut-Katanga et Lualaba) ; d'élaborer et de mettre en œuvre un plan d'aménagement du territoire pour éviter des constructions anarchiques qui causent les gîtes larvaires; de renforcer les 
projets d'assainissements en milieux urbain et rural ; d'initier des enquêtes entomologiques dans chaque zone de dénombrement lors des enquêtes démographiques de santé (EDS) et de s'intéresser à la spatialisation des données pour identifier les déterminants environnementaux du paludisme.

\section{References:}

1. Abdou Louche (2014), Évolution spatio-temporelle de la morbidité palustre chez les enfants de moins de cinq ans au Niger, mémoire de Master en Démographie, Université de Yaoundé II Soa, IFORD, 144 p. + annexes.

2. Association Française Des Enseignants De Parasitologie Et Mycologie (2014), Paludisme, France, Document de travail, Paris, Université Médicale Virtuelle Francophone, 27p.

3. AUBRY Pierre Et GAÜZERE Bernard-Alex (2018), Paludisme, Document pédagogique, Centre René Labusquière, Institut de Médecine Tropicale, Université de Bordeaux, 30p.

4. Barbieri M. (1991), Les déterminants de la mortalité des enfants dans le tiers monde, Les dossiers du CEPED, Paris, $\mathrm{n}^{\circ} 18,35 \mathrm{p}$.

5. Barry Mamadou Saliou (2015), Disparités régionales de la morbidité palustre chez les enfants de moins de cinq ans en Guinée, mémoire de Master en Démographie, Université Yaoundé II Soa, IFORD, 140 p. tannexes.

6. Banque Mondiale (2018), Diagnostic systématique pays : Priorités de politiques pour réduire la pauvreté et promouvoir la prospérité partagée dans un pays fragile sortant d'un conflit, République démocratique du Congo, Document de travail, Kinshasa, $\mathrm{n}^{\circ} 112733$ ZR, 188p.

7. Boco Germain (2011), Déterminants individuels et contextuels de la mortalité des enfants de moins de cinq ans en Afrique au sud du Sahara : Analyse comparative des enquêtes démographiques et de santé, Thèse en Démographie, Université de Montréal, 249p + annexes.

8. Bouba Djourdebbe Franklin (2015), Facteurs environnementaux immédiats et santé des enfants dans les zones de l'Observatoire de population de Ouagadougou, thèse en Démographie, Université de Montréal, 210p. + annexes.

9. Capelle Angélique (2007), Paludisme et réchauffement climatique, mémoire de 2è année en Ecologie Humaine et Santé, Université Paul Cézanne Aix-Marseille, 28p. + annexes.

10. Carnevale P. Et Robert V. (2009), Les anophèles: Biologie, transmission du Plasmodium et lutte anti-vectorielle, Marseille (France), IRD Éditions, 402p.). 
11. Carnevale P. Et Vaugelade J. (1987), Paludisme, morbidité palustre et mortalité infantile et juvénile en Afrique sub-saharienne, WHO/MAL/87.1036, Document de travail, $20 \mathrm{p}$.

12. Courgeau D. Et Baccaïni B. (1997), «Analyse multi-niveaux en sciences sociales », population, vol.4, pp.831-863.

13. Diallo D. (2016), Disparités régionales de la morbidité palustre chez les enfants de 6-59 mois au Mali : Recherche des facteurs explicatifs, mémoire de Master en Démographie, Université Yaoundé II Soa, IFORD, 95p. + annexes.

14. Diallo Yveline. (2003). «Pauvreté et maladie », in : Jaffré Y. et Olivier de Sardan J. (2003), Une médecine inhospitalière : les difficiles relations entre soignants et soignés dans cinq capitales d'Afrique de l'Ouest, Marseille (France), Editions Hommes et Société, pp.157-216.

15. Doumbia Sidy (2007), Impact du changement climatique sur l'incidence du paludisme au Mali de 1998 à 2007, thèse en Médecine, Université de Bamako, 86p.

16. Drame Papa Makthar (2010), Relation Homme-Vecteur au cours du paludisme: Développement d'un nouvel outil immunoépidémiologique évaluant l'éfficacité de la lutte anti-vectorielle, thèse en Biologie et pathologies humaines, Université Cheik Anta Diop, Ecole Doctorale «Sciences de la vie, de la santé et de l'environnement », 346p. + annexes.

17. Fournet F. et al. (2015), «Analyse de la distribution socio-spatiale du paludisme dans une ville moyenne ouest africaine, Bobo-Dioulasso (Burkina Faso)», Dynamiques Environnementales-Journal international des géosciences et de l'environnement, $\mathrm{n}^{\circ} 36, \mathrm{pp} .155-$ 169.

18. Fournier P. Et Haddad S. (1995), « Les facteurs associés à l'utilisation des services de santé dans les pays en développement », in : Hubert G. Et Piche V.(1995), Sociologie des populations, Montréal, PUM/AUPELF-UREF, pp 289-325.

19. Franckel Aurélien (2004), Les comportements de recours aux soins en milieu rural au Sénégal: Le cas des enfants fébriles à Niakhar, thèse de doctorat en Démographie, Université Paris X Nanterre, 466p. + annexes.

20. Givord Pauline Et Guillerm Marine (2016), Méthodologie statistique : Modèles multiniveaux, INSEE, Document de travail, Paris, 64p.

21. Hajison P. et al. (2018), "Malaria in children under-five: A comparison of risk factors in lakeshore and highland areas, Zomba district, Malawi », PLoS ONE, Vol. 13, n¹1, pp. 1-16. 
22. Hanitriniaina Felana Angella Ihantamalala (2017), Modélisation de la dynamique du paludisme à Madagascar, thèse en Géographie, Université de la réunion, 120p + annexes.

23. INS-RDC (2014), Rapport global de l'enquête 1-2-3: Résultats de l'enquête sur l'emploi, le secteur informel et sur la consommation des ménages de 2012, Rapport de l'enquête, Kinshasa, 164p.

24. INS-RDC (2018), Enquête par grappes à indicateurs multiples de 2017-2018, République Démocratique du Congo, Rapport de résultats de l'enquête, Kinshasa, 601p.

25. Itriago Deborah (2011), Décider de son développement: La fiscalité pour combattre la pauvreté, Rapport de recherche d'OXFAM, Londres, 8p.

26. Kabore Sidbewende Théodore (2019), Disparités régionales de la morbidité palustre des enfants de moins de cinq ans au Burkina Faso, mémoire de Master en démographie, Université de Yaoundé II SOA, IFORD, $175 \mathrm{p}+$ annexes.

27. Kone Karna Géorges (2012), L'équité de l'accès aux soins dans un contexte de subvention des médicaments : Une analyse économétrique des déterminants du recours aux soins à Dakar, thèse de doctorat en économie de la santé, Université Cheikh Anta Diop, 239p. + annexes.

28. Le Hesran J. (2000), «Les particularités du paludisme chez l'enfant », Med Trop, Vol. 60, n 1 , pp. 92-98.

29. Longuepee D. (2006), «Paludisme, institutions et croissance: Que penser du débat actuel ? ", Économie et institutions, n ${ }^{\circ}$, pp. 95-118.

30. Losimba Joris, Emina Jacques, Mbolipay Josué, Karamere Johanna, Kakesa Olivier, Nana Ismaël, Humes Michael Et Ye Yazouma (2018), Evaluation de l'impact des interventions de lutte contre le paludisme sur la mortalité toutes causes confondues chez les enfants de moins de cinq ans en République Démocratique du Congo de 2005 à 2015, PnlpRdc, Document de travail, 77p.

31. Makamba Mbonariba Et Manunga (2004), La problématique du financement de la sante en République Démocratique du Congo, Document de travail, Ministère de la santé, Kinshasa, 27p.

32. Mamadou Lamine Poreko (2018), Etudiants subsahariens à Toulouse et prévention du paludisme d'importation, thèse en Médecine, Université Toulouse III-Paul SABATIER, 71p. + annexes.

33. Maud Cussac (2006), Impacts des facteurs environnementaux et sociodémographiques dans la ville d'Ouagadougou (Burkina Faso), Mémoire de Master en Géographie de la santé, Université de Nanterre, $58 \mathrm{p}$. + annexes.

34. Moiroux Nicolas (2012), Modélisation du risque d'exposition aux moustiques vecteurs de Plasmodium spp. Dans un contexte de lutte 
anti-vectorielle, thèse en Ecologie, Environnement, Université Montpellier II, 252 p. + annexes.

35. Ministere Du Plan Et Suivi Des Mise En CEuvre De La Revolution De La Modernite, Ministere De La Sante Publique, et ICF INTERNATIONAL (2014), Enquête Démographique et de Santé, République Démocratique du Congo 2013-2014, Calverton, Maryland, U.S.A. : MPSMRM, MSP et ICF International, $678 \mathrm{p}$.

36. Ministere De La Sante Publique (2016), Plan National de développement Sanitaire (PNDS) 2016-2020: Vers la couverture sanitaire universelle, MSP, Kinshasa, 97p.

37. Mutombo A. et al. (2018), «Paludisme grave chez les enfants âgés de moins de cinq ans à l'hôpital Panda à Likasi, République Démocratique du Congo », Revue de l'Infirmier Congolais, Vol. 2, pp. 4-10.

38. Nganawara D. (2016), Famille et scolarisation des enfants en âge obligatoire scolaire au Cameroun : Une analyse à partir du recensement de 2005 (Collection Rapport de recherche de l'Observatoire Démographique et Statistique de l'Espace Francophone (ODSEF), Université Laval, Québec, 50p.

39. Nkemba B. et al. (2014), «Vulnérabilité économique des ménages au cours d'une épisode du paludisme dans la zone de santé de MitiMurhesa en RD Congo », International Journal of Innovation and Applied Studies, Vol. 8, n³, pp. 912-919.

40. Noguer A. (1979), «Les facteurs influençant la distribution géographique du paludisme dans le monde", Revue genevoise de géographie (Globe), Tome 119, pp. 15-25.

41. ORGANISATION MONDIALE DE LA SANTE (2015), Rapport mondial sur le paludisme 2015, Suisse, Document de travail, Genève, $165 \mathrm{p}$.

42. ORGANISATION MONDIALE DE LA SANTE (2018), Rapport mondial sur le paludisme 2017, Suisse, Document de travail, Genève, $196 \mathrm{p}$.

43. Othingue N. (2005), Étude épidémiologique et spatiale du paludisme en milieu urbain au Sahel: N'Djaména, Tchad, thèse de Doctorat en Géographie, Université de Bâle (Suisse), 156 p. + annexes.

44. Ouedraogo M. et al. (2018), «Spatial distribution and determinants of asymptomatic malaria risk among children under five years in 24 districts in Burkina Faso », Malaria Journal, Vol. 17, n460, pp. 1-12.

45. Pierrat Charlotte (2010), Des moustiques et des hommes, thèse de en Géographie, Université Paris 1 Panthéon Sorbonne, 149p + annexes.

46. PNLP-RDC (2017), Rapport annuel d'activités 2016, République Démocratique du Congo, Rapport, Kinshasa, 44p. 
47. PNUD (2009a), Kinshasa : Pauvreté et conditions de vie des ménages, République Démocratique du Congo, Document de travail, Kinshasa, 20p.

48. PNUD (2009b), Kasaï Occidental : Pauvreté et conditions de vie des ménages, Document de travail, Kasaï Occidental, 20p.

49. PNUD (2009c), Kasaï Oriental : Pauvreté et conditions de vies ménages, Document de travail, Kasaï Oriental, 20p.

50. PNUD (2009d), Katanga : Pauvreté et conditions de vie des ménages, Document de travail, Katanga, 20p.

51. Rakotondrabe Faraniaina Patricia (2004), Statut de la femme et santé des enfants à Madagascar, thèse de Doctorat en Démographie, Université de Yaoundé II Soa, IFORD (Cameroun), 370 p. + annexes.

52. Remy G. (1988), «Spécificités urbaines du paludisme en Afrique tropicale ». ORSTOM, série Entomo. Méd, Vol. 6, n², pp. 3-20.

53. SabatinellI G. et al. (1986), «Prévalence du paludisme à Ouagadougou et dans le milieu rural limitrophe en période de transmission maximale », Parassitologia, Vol. 28, n ${ }^{\circ}$, pp. 17-31.

54. Saïdou Hamadou (2018), Pauvreté, paludisme et réformes des systèmes de santé en Afrique: Trois études appliquées au Cameroun, thèse en sciences économiques, Université Paris-Dauphine, 181p. + annexes.

55. Samakou Guy Landry (2017), Les facteurs individuels et contextuels de la morbidité palustre infanto-juvenile au Bénin, mémoire de Master en démographie, Université de Yaoundé II SOA, IFORD, 93p. + annexes.

56. Soma D. et al. (2018), «Transmission entomologique du paludisme à Nanoro, site de l'essai vaccinal RTS'S au Burkina Faso », Revue du CAMES: Science de la santé, Vol. 5, n², pp. 15-20.

57. Stefani Aurélien (2011), Epidémiologie du paludisme et environnement: Étude de deux populations amérindiennes de l'est et de l'ouest guyanais, thèse pour le doctorat en Sciences de la Vie, Spécialité Santé Publique, Université des Antilles et de la Guyane, 369p. + annexes.

58. Takudzwa David, (2018), Household determinants of malaria in Mutasa District of Zimbabwe, Thesis in Public Health, Walden University, $172 p+$ appendix.

59. UNICEF, O. D. N. U. P. L. (2018). Kasaï: Les enfants, premières victimes de la crise. Rapport, Kinshasa, 24p.

60. UNITED STATES AGENCY FOR INTERNATIONAL DEVELOPMENT (2014), Comprendre et Utiliser les Enquêtes Démographiques et de Santé, U.S.A., Document de travail, Calverton, $65 \mathrm{p}$. 
61. Yovoga R. (2009), Recours thérapeutiques des mères en cas de paludisme chez les enfants de moins de cinq ans au Bénin, mémoire de Master en Démographie, Université de Yaoundé II Soa, IFORD (Cameroun), 154p. + annexes.

\section{Annexe}

Annexe 1. Taux de non-réponse des variables de l'étude

\begin{tabular}{|c|c|c|c|c|c|}
\hline Variables & Modalités de la variable & $\begin{array}{l}\text { \% de la } \\
\text { modalit } \\
\text { é }\end{array}$ & $\begin{array}{l}\text { Répons } \\
\text { es } \\
\text { valides }\end{array}$ & $\begin{array}{l}\text { Valeurs } \\
\text { manquant } \\
\text { es }\end{array}$ & $\%$ \\
\hline \multirow{2}{*}{$\begin{array}{l}\text { Résultat de la goutte } \\
\text { épaisse }\end{array}$} & Impaludé & 26,34 & 8489 & 0 & 0,00 \\
\hline & Non impaludé & 73,66 & & & \\
\hline \multirow[t]{2}{*}{ Sexe de l'enfant } & Masculin & 49,84 & 8489 & 0 & 0,00 \\
\hline & Féminin & 50,16 & & & \\
\hline \multirow[t]{2}{*}{ Age de l'enfant } & $6-23$ mois & 32,84 & 8489 & 0 & 0,00 \\
\hline & $24-59$ mois & 67,16 & & & \\
\hline \multirow{2}{*}{$\begin{array}{l}\text { Utilisation de la } \\
\text { moustiquaire }\end{array}$} & N'utilise pas & 45,54 & 8489 & 0 & 0,00 \\
\hline & Utilise & 54,46 & & & \\
\hline \multirow[t]{3}{*}{ Age de la mère } & $15-24$ ans & 40,07 & 8088 & 401 & 4,72 \\
\hline & $25-34$ ans & 43,45 & & & \\
\hline & $35-49$ ans & 16,48 & & & \\
\hline \multirow[t]{7}{*}{ Ethnie de la mère } & Bakongo & 6,56 & 8046 & 443 & 5,21 \\
\hline & Bas-Kasaï et Kwilu-Kwango & 15,29 & & & \\
\hline & Cuvette centrale & 9,66 & & & \\
\hline & Ubangi et Imbiri & 13.44 & & & \\
\hline & Uélé-Lac Albert & 8,8 & & & \\
\hline & Basele-Komo-Maniema et Kivu & 17,09 & & & \\
\hline & Kasaï-Katanga et Tanganyika & 29,17 & & & \\
\hline \multirow[t]{3}{*}{ Religion de la mère } & Catholique & 43.22 & 8059 & 430 & 5,07 \\
\hline & Protestante & 27,35 & & & \\
\hline & Autre religion & 29,43 & & & \\
\hline \multirow{3}{*}{$\begin{array}{l}\text { Niveau d'instruction } \\
\text { de la mère }\end{array}$} & Sans niveau & 21,80 & 7725 & 764 & 9,00 \\
\hline & Primaire & 44,89 & & & \\
\hline & Secondaire et plus & 33,31 & & & \\
\hline \multirow[t]{3}{*}{$\begin{array}{l}\begin{array}{l}\text { Occupation de la } \\
\text { mère }\end{array} \\
\end{array}$} & Inactive & 24,04 & 8088 & 401 & 4,72 \\
\hline & Agricultrice & 52,27 & & & \\
\hline & Active non agricultrice & 23,69 & & & \\
\hline \multirow{3}{*}{$\begin{array}{l}\text { Culture du ménage } \\
\text { Paludisme }\end{array}$} & Faible & 19,89 & 8482 & 7 & 0,08 \\
\hline & Moyenne & 48,21 & & & \\
\hline & Forte & 31,90 & & & \\
\hline Niveau de vie du & Bas & 40,00 & 8387 & 102 & 1,20 \\
\hline
\end{tabular}




\begin{tabular}{|c|c|c|c|c|c|}
\hline \multirow[t]{2}{*}{ ménage } & Moyen & 40,00 & & & \\
\hline & Elevé & 20,00 & & & \\
\hline \multirow{3}{*}{$\begin{array}{l}\text { Promiscuité dans le } \\
\text { ménage }\end{array}$} & $1-2$ enfants & 20,18 & 8489 & 0 & 0,00 \\
\hline & 3 enfants & 34,39 & & & \\
\hline & Plus de 3 enfants & 45,43 & & & \\
\hline \multirow[t]{2}{*}{ Type de toilettes } & Toilette non améliorée & 62,94 & 8488 & 1 & 0,01 \\
\hline & Toilette améliorée & 37,06 & & & \\
\hline \multirow{3}{*}{$\begin{array}{l}\text { Disponibilité de la } \\
\text { Moustiquaire }\end{array}$} & Aucune moustiquaire & 25,72 & 8489 & 0 & 0,00 \\
\hline & $\begin{array}{l}\text { Une moustiquaire pour plus de } \\
\text { deux }\end{array}$ & 10,70 & & & \\
\hline & Une moustiquaire pour deux & 63,59 & & & \\
\hline \multirow[t]{2}{*}{ Milieu de résidence } & Rural & 70,88 & 8489 & 0 & 0,00 \\
\hline & Urbain & 29,12 & & & \\
\hline \multirow{6}{*}{$\begin{array}{ll}\text { Province } & \mathrm{de} \\
\text { résidence } & \end{array}$} & Ouest & 22,95 & 8489 & 0 & 0,00 \\
\hline & Nord-ouest & 15,88 & & & \\
\hline & Centre & 20,56 & & & \\
\hline & Sud-est & 12,59 & & & \\
\hline & Est & 16,26 & & & \\
\hline & Nord-est & 11,70 & & & \\
\hline \multirow{2}{*}{$\begin{array}{l}\text { Proportion } \mathrm{de} \\
\text { ménages pauvres }\end{array}$} & Elevée & 39,26 & 8489 & 0 & 0,00 \\
\hline & Faible & 60,74 & & & \\
\hline
\end{tabular}

Source : Exploitation des données de l'EDS-RDC II, 2013-2014

Annexe 2. Variables incluses dans l'indicateur niveau de vie du ménage

\begin{tabular}{|l|l|l|l|}
\hline Variables & $\begin{array}{l}\text { Réponses } \\
\text { valides }\end{array}$ & $\begin{array}{l}\text { Réponses } \\
\text { manquantes }\end{array}$ & $\begin{array}{l}\text { Taux de non } \\
\text { réponses en \% }\end{array}$ \\
\hline Confort de l'habitat & & & \\
\hline - Source d'eau de boisson & 8487 & 2 & 0,02 \\
\hline -Matériau du sol & 8484 & 5 & 0,06 \\
\hline -Principal matériau du toit & 8488 & 1 & 0,01 \\
\hline -Principal matériau des murs extérieurs & 8484 & 5 & 0,06 \\
\hline Possessions de certains biens & & & \\
\hline -Possession des biens matériels & & & \\
\hline - Electricité & 8484 & 5 & 0,06 \\
\hline - Poste radio & 8484 & 5 & 0,06 \\
\hline -Télévision & 8485 & 4 & 0,05 \\
\hline - Réfrigérateur & 8488 & 1 & 0,01 \\
\hline -Générateur & 8487 & 2 & 0,02 \\
\hline - Réchaud & & & \\
\hline - Chaises & 8484 & 5 & 0,06 \\
\hline - Lits & 8483 & 6 & 0,07 \\
\hline - Lampes & 8486 & 3 & 0,04 \\
\hline - Four & 8470 & 19 & 0,22 \\
\hline - Machine à coudre & 8477 & 12 & 0,14 \\
\hline - Bicyclette & 8485 & 4 & 0,05 \\
\hline
\end{tabular}




\begin{tabular}{|l|l|l|l|}
\hline - Téléphone portable & 8487 & 2 & 0,02 \\
\hline - Motocyclette & 8487 & 2 & 0,02 \\
\hline - Voiture & 8487 & 2 & 0,02 \\
\hline - Ordinateur & 8487 & 2 & 0,02 \\
\hline - Loyer & 8481 & 8 & 0,09 \\
\hline
\end{tabular}

Source : Exploitation des données de l'EDS-RDC II, 2013-2014

Annexe 3. Variables incluses dans l'indicateur Culture de la mère sur le paludisme

\begin{tabular}{|l|l|l|l|}
\hline Variables & $\begin{array}{l}\text { Réponses } \\
\text { valides }\end{array}$ & $\begin{array}{l}\text { Réponses } \\
\text { manquantes }\end{array}$ & $\begin{array}{l}\text { Taux de non } \\
\text { réponse en \% }\end{array}$ \\
\hline Comment attrape-t-on le paludisme & 8484 & 5 & 0,06 \\
\hline - Piqûre de moustique & 8484 & 5 & 0,06 \\
\hline - Piqûre autres insectes & 8484 & 5 & 0,06 \\
\hline - Transfusion sanguine & 8484 & 5 & 0,06 \\
\hline - De la mère à l'enfant & 8484 & 5 & 0,06 \\
\hline - Eau à boire & 8484 & 5 & 0,06 \\
\hline - sorcellerie & \multicolumn{2}{l|}{} \\
\hline Les symptômes du paludisme & 8486 & 3 & 0,04 \\
\hline - Fièvre & 8486 & 3 & 0,04 \\
\hline - Maux de tête & 8486 & 3 & 0,04 \\
\hline - Douleur généralisée & 8486 & 3 & 0,04 \\
\hline - Diarrhée & 8486 & 3 & 0,04 \\
\hline - Coma & 8486 & 3 & 0,04 \\
\hline - Frisson & 8486 & 3 & 0,04 \\
\hline - Convulsion & \multicolumn{2}{l|}{} \\
\hline Comment se protéger contre les piqûres des moustiques & 8484 & 5 & 0,06 \\
\hline - Utilisation de l'insecticide & 8484 & 5 \\
\hline - Dormir sous une MII & 8484 & 5 & 0,06 \\
\hline - Dormir sous une moustiquaire simple & 8484 & 5 & 0,06 \\
\hline - Assainissement &
\end{tabular}

Source : Exploitation des données de l'EDS-RDC II, 2013-2014 